\title{
The role of behavioural flexibility in primate diversification
}

Maria J.A. Creighton ${ }^{\mathrm{a}}$, Dan A. Greenberg ${ }^{\mathrm{b}}$, Simon M. Reader ${ }^{\mathrm{a}} \&$ Arne Ø. Mooers ${ }^{\mathrm{b}}$

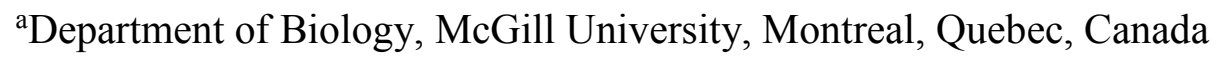

${ }^{b}$ Department of Biological Sciences, Simon Fraser University, Burnaby, British Columbia, Canada

*Author for correspondence: maria.creighton@mail.mcgill.ca; ph: +1-514 398 7020; McGill University, Stewart Biology Building 1205 ave Docteur Penfield, Montreal, QC Canada, H3A 1B1 ORCID:

https://orcid.org/0000-0003-1337-1946

DECLARATIONS OF INTEREST: None 
1 Identifying the factors that influence species diversification is fundamental to our understanding

2 of the evolutionary processes underlying extant biodiversity. Behavioural innovation, coupled

3 with the social transmission of new behaviours, has been proposed to increase rates of

4 evolutionary diversification, as novel behaviours expose populations to new selective regimes.

5 Thus, it is believed that behavioural flexibility may play an important role in driving

6 evolutionary diversification across animals. We test this hypothesis within the primates, a

7 taxonomic group with considerable among-lineage variation in both species diversity and

8 behavioural flexibility. We employ a time cut-off in our phylogeny to help account for biases

9 associated with recent taxonomic reclassifications and compare three alternative measures of

10 diversification rate that consider different phylogenetic depths. We find that the presence of

11 behavioural innovation and social learning are positively correlated with diversification rates

12 among primate genera, but not at shallower phylogenetic depths. Given that we find stronger

13 associations when examining older rather than more recent diversification events, we suggest

14 that extinction resistance, as opposed to speciation, may be an important mechanism linking

15 behavioural flexibility and primate diversification. Our results contrast with work linking

16 behavioural flexibility with diversification of birds at various phylogenetic depths. We offer a

17 possible dispersal-mediated explanation for these conflicting patterns, such that the influence

18 behavioural flexibility plays in dictating evolutionary trajectories differs across clades. Our

19 results suggest that behavioural flexibility may act through several different pathways to shape

20 the evolutionary trajectories of lineages. 
23 KEYWORDS: behavioural drive; behavioural flexibility; evolutionary diversification;

24 extinction; primates; speciation

26 Extant species diversity is remarkably variable across the Tree of Life (Willis, 1922; Williams,

27 1964). For clades of the same age, differences in net diversification rate (i.e. speciation rate

28 minus extinction rate) ultimately drive differences in clade size. Both the external environment

29 (e.g. Badgley, 2010; Kozak \& Wiens, 2010; Frey, 2010) and intrinsic features of a lineage (e.g.

30 Raikow, 1986; Heard \& Hauser, 1995) can influence net diversification. Despite ongoing study,

31 there remains considerable debate over the factors that lead to differences in diversification rate

32 among lineages, and their relative importance (e.g. Lewontin, 1983; West-Eberhard, 1989; Isaac,

33 et al., 2005; Rabosky, 2009; see review by Wiens, 2017).

35 Plasticity has been repeatedly proposed to play a major role in shaping evolutionary trajectories

36 in general, and speciation in particular (Baldwin, 1902; Lewontin, 1983; Bateson, 1988; Wcislo,

37 1989; Odling-Smee, et al., 2003; West-Eberhard, 2003; Pelletier, et al., 2009; also see review in

38 Pfennig, et al., 2010), and theoretical modelling supports its potential influence (e.g. Hinton \&

39 Nowlan, 1987; Anderson, 1995; Behera \& Nanjundiah, 1995; Ancel, 1999; 2000). Behavioural

40 development and expression often allow for more rapid responses than other forms of plasticity

41 such as induced morphological changes (Duckworth, 2009; Snell-Rood, 2013; West-Eberhard,

42 2003). Thus, plasticity of behaviour has been hypothesized to play a particularly important role

43 in influencing evolutionary trajectories of animal lineages (Wyles, et al., 1983; Wilson, 1985;

44 West-Eberhard, 2003). The propensity to adopt new behaviours can greatly and quickly alter

45 ecological niches, exposing populations to new selective regimes. This can lead to increased trait 
46 disparity among populations (the "behavioural drive hypothesis"; Wyles, et al., 1983; Wilson,

47 1985); importantly, divergent selection can also lead to eventual speciation. As a result, it has

48 been suggested that behaviourally flexible taxa (i.e. those taxa exhibiting high propensities for

49 behavioural change due to, for example, learning or readiness to transition to new conditions; Sol

50 \& Lefebvre, 2000; Audet \& Lefebvre, 2017) may undergo faster rates of speciation, and thus

51 overall net rates of evolutionary diversification, compared to less flexible taxa (Sol, et al., 2005;

52 Grant \& Grant, 2008; Price, 2008; Sol \& Price, 2008; Tebbich, et al., 2010). However, despite

53 support from theoretical modelling (e.g. Price, et al., 2003; Lachlan \& Servedio, 2004; Lapiedra,

54 et al., 2013), the idea that behavioural flexibility enhances diversification rates remains

55 contested. Some dispute the extent to which behaviour plays an active role in dictating animal

56 diversity (e.g. Scott-Phillips, et al., 2014). Meanwhile, an alternative hypothesis posits that

57 behavioural flexibility may inhibit, rather than enhance, species diversification: populations that

58 can utilize new resources or transition to new environments are shielding their genomes from

59 bouts of strong directional selection (Bogert, 1949; Huey, et al., 2003).

60

61 Duckworth (2009) suggests that behavioural flexibility could both dampen and promote

62 evolutionary rates depending on the time scale. Under this proposed framework, behavioural

63 flexibility inhibits evolution on short time scales by buffering against abrupt environmental

64 changes that may otherwise result in a population bottleneck or strong bouts of directional

65 selection. Over longer time scales, the same behavioural shift can lead to speciation, either by

66 setting the stage for allopatric speciation or by exposing the newly situated population to novel

67 selective regimes (Huey, et al., 2003; Losos, et al., 2004; Duckworth, 2009; see example in

68 Muñoz \& Losos, 2018). 
70 Previous studies have provided support for behavioural flexibility both driving (e.g. Yeh, 2004;

71 Yeh \& Price, 2004; Tebbich, et al., 2010, Riesch, et al., 2012; Foote, et al., 2016) and inhibiting

72 (e.g. Losos, et al., 2004; Weber, et al., 2004; Shultz, et al., 2005; Gonzalez-Voyer, et al., 2016)

73 evolution. However, many of these studies have primarily considered the effects of behavioural

74 flexibility on microevolutionary change at short time scales (e.g. recent speciation events or

75 population decline). One notable exception is in birds, where behavioural flexibility has been

76 associated with multiple estimates of macroevolutionary lineage diversification (Nicolakakis, et

77 al., 2003; Sol, et al., 2005 - also see Sol, 2003; Sayol, et al., 2019). These studies have employed

78 two proposed correlates of behavioural flexibility: brain size relative to body size (a structural

79 measure presumed and shown elsewhere to be associated with behavioural flexibility; e.g.

80 Lefebvre, et al., 2004) and innovation rate (a behavioural measure) taken from literature surveys.

81 Both large relative brain size and high innovation rates were associated with heightened

82 diversification in birds (Nicolakakis, et al., 2003; Sol, et al., 2005; Sayol, et al., 2019), consistent

83 with the idea that behavioural flexibility positively impacts diversification. Importantly, such

84 tests have yet to be applied across other taxa, making it difficult to generalize the role of

85 behaviour in shaping the Tree of Life.

87 Here, we explore the relationship between four proxies of behavioural flexibility and several

88 measures of diversification rate in primates, a taxonomic group with considerable among-lineage

89 variation in both behavioural flexibility (Reader \& Laland, 2002; Reader, et al., 2011) and extant

90 species diversity (Upham, et al., 2019a; 2019b). Variables associated with diversity of other taxa

91 (e.g. geographic range size and latitude) have been shown to be poor predictors of primate 
92 diversification (Arbour \& Santana, 2017; Upham, et al., 2019a), leaving a great deal of what

93 shapes extant primate diversity unexplained. We focus on two behavioural measures of

94 behavioural flexibility, the presence or absence of published reports of innovation and of social

95 learning, and two brain size measures widely thought to be associated with ability to exhibit

96 flexible behaviours. Consistent with what has been reported for birds (Nicolakakis, et al., 2003;

97 Sol, et al., 2005; Sayol, et al., 2019) and Duckworth (2009)'s proposal that behavioural

98 flexibility promotes diversification events over longer evolutionary time scales, we predict that

99 our separate measures of behavioural flexibility will covary positively with diversification rates

100 across primate lineages. To examine how this association changes at different phylogenetic

101 depths, we examine how behavioural flexibility correlates with diversification over both

102 shallower and deeper time depths in our phylogeny. Understanding how behaviour and ecology

103 may interact to shape evolutionary patterns provides a glimpse into some of the processes that

104 have shaped primate biological diversity and could, in turn, dictate future diversity.

106 METHODS:

107 Data

108 Diversification Rate

109 Estimating diversification rates is challenging because it depends on an accurate assessment of

110 both the taxonomic richness and divergence time of a given lineage. A previous study testing

111 whether behavioural flexibility drives shallow divergences used subspecies per species as a

112 measure of subspecific diversification in birds (Sol, et al., 2005). Using this subspecies metric

113 could introduce considerable bias when applied in primates, however, as primate taxonomic 
114 richness has changed drastically over recent decades (Tattersall, 2007; Groves, 2014; Rylands \&

115 Mittermeier, 2014), with much of this change attributed to application of the 'phylogenetic

116 species concept' (PSC) and its tendency to raise former subspecies and variants to the full

117 species rank (Tattersall, 2007). Importantly, it has been suggested that these elevations in

118 subspecies status have been biased toward certain taxa (Isaac, et al., 2004), which would lead to

119 inconsistent estimates of species versus subspecies richness across lineages. Studies using other

120 estimates of primate diversification (i.e. diversification analyses using TreePar; Stadler, 2011)

121 have been hindered by the applications of the PSC, particularly when it comes to accurately

122 estimating shallow divergences (Springer, et al., 2012). Modern primate phylogenies are also not

123 reflective of modern primate taxonomies, as they include some phylogenetic species and omit

124 others, preventing us from using recently described evolutionary rate measures (see, e.g., Jetz, et

125 al., 2012; Mitchell \& Rabosky, 2017) that rely on a comprehensive phylogeny with consistent

126 species designations among clades. Instead, we used well-resolved "lineages" that putatively

127 reflect stable species complexes. We started with the most widely used, dated primate tree

128 publicly available at the time of this study, the GenBank taxonomy consensus tree provided on

129 the 10kTrees website (version 3) (Arnold, et al., 2010), containing 301 tips. We then created a

130 time cut-off in the tree at the time when we determined a majority of robust biological species

131 described in Honacki et al. (1982) had evolved (1.1mya). We chose Honacki et al. (1982) as it

132 was the last major primate taxonomy published before the introduction of the PSC (Cracraft,

133 1983). After creating this time cut-off, we subsequently eliminated shallow divergences

134 occurring after 1.1mya from the consensus tree (see Figure S1). Each branch in the tree that was

135 extant at 1.1mya was retained in the tree and designated as a stable "lineage". We then

136 additionally pruned species from this phylogeny that were no longer recognized by modern 
137 taxonomies (IUCN/SSC Primate Specialist Group, 2018). This resulted in 241 identifiable

138 lineages to compare in terms of taxonomic richness and divergence times. Using the most

139 recently published primate species and subspecies list from the IUCN/SSC Primate Specialist

140 Group (2018), we referenced taxonomic and phylogenetic works to assign each of the 705

141 species and subspecies to one of these 241 lineages. This allowed us to assign each lineage an

142 agnostic "taxon richness" score (i.e. the sum of all monotypic species and subspecies in a

143 lineage; see Figure S1) that accounts for the discrepancies in subspecies elevations across

144 lineages. Each species or subspecies listed by the IUCN/SSC Primate Specialist Group (2018)

145 was also assigned to one of the 66 genera named in our 10kTrees phylogeny (Arnold, et al.,

146 2010). After eliminating two individual species and one genus in our tree that could not be

147 resolved using these methods (see supplementary material), our study considered 703 taxa

148 (species or subspecies) assigned to 239 lineages and 65 genera.

150 To estimate lineage diversification rate we used the method-of-moments estimator (Magallon \&

151 Sanderson, 2001) that divides the natural log of "taxon richness" (species and subspecies) by

152 lineage stem age to produce a 'Taxa per Lineage Diversification Rate' that should be less biased

153 by recent subspecies elevations. This method was repeated at the genus-level where the natural

$154 \log$ of "taxon richness" for each genus was divided by the stem age of that genus (hereafter

155 'Taxa per Genus Diversification Rate'). We note that all log transformations referenced hereafter

156 refer to natural $\log$ transformations $\left(\log _{\mathrm{e}}\right)$. Lastly, we created a second, and perhaps more

157 conservative estimate of genus diversification rate where richness scores were generated using

158 the number of lineages $(n=239)$ per genus, rather than the number of taxa described by the

159 IUCN/SSC Primate Specialist Group (2018), hereafter 'Lineage per Genus Diversification Rate'. 
160 A few genera were not monophyletic on our tree, and we considered these on a case-by-case

161 basis ultimately removing five from our genus-level analysis (see the supplementary material).

163 We illustrate these methods and present associated calculations using genus Aotus as an example

164 in the supplementary material to provide some considerations on the potential uses and

165 limitations of this approach.

\section{Behavioural Proxies of Behavioural Flexibility}

168 We focused on two key behaviours to infer behavioural flexibility: innovation and social

169 learning. Innovation and social learning are both important in determining the macroevolutionary

170 effects of behavioural flexibility because multiple individuals must acquire an innovation

171 through social learning or independent innovation to have population-level effects (Wyles, et al.,

172 1983; Wilson, 1985; Duckworth, 2009). In addition to facilitating the transmission of

173 innovations throughout a population, social learning can also be a valuable measure of

174 population-level behavioural flexibility on its own as it reflects the ability of individuals within a

175 population to pick up behaviours that are novel to them but not necessarily novel to the

176 population. We note that social learning and innovation are taxonomically widespread (Reader \&

177 Biro, 2010; Snell-Rood, et al., 2015). However, we assume that species with no published

178 accounts of social learning or innovation are likely relying on these behaviours infrequently.

179 Moreover, the innovation and social learning data used here have been positively associated with

180 other measures of behavioural flexibility (e.g. brain size measures; Reader, et al., 2011;

181 Navarrete, et al., 2016). For innovation, we focus on technical innovations (classified as those 
182 involving tool use following Navarrete, et al., 2016) because these more easily defined

183 behaviours have been linked to complex cognition (Overington, et al., 2009), and reports of other

184 classes of innovation (e.g. food type innovation) can be highly influenced by opportunistic

185 events (Ducatez, et al., 2015). Combined with the fact that taxa with reports of technical

186 innovation also tended to be those with evidence of other innovation types (see data in Navarrete,

187 et al., 2016), this likely makes technical innovation a robust estimate of innovativeness across

188 primates.

189

190 Counts of innovation and social learning per lineage came from Reader et al. (2011) and

191 Navarrete et al. (2016). Reader et al. (2011) established this dataset through a survey of over

1924000 published articles for examples of social learning and behavioural innovation. Reader et al.

193 (2011, p. 1018) define an innovation as the tendency to "discover novel solutions to

194 environmental or social problems". These data were later subdivided into different innovation

195 categories by Navarrete et al. (2016), including 'technical' innovations, defined as innovations

196 involving tool use. Reader et al. (2011, p. 1018) define social learning as the tendency to "learn

197 skills and acquire information from others", including instances of social learning from both kin

198 and unrelated individuals. Social learning was often inferred from observational data in the

199 original reports.

201 As an alternative to treating behavioural data as a binary metric (e.g. presence or absence of

202 innovativeness in Ducatez, et al., 2020) some studies have used "rate" measures of behaviours:

203 residuals from a $\log -\log$ plot of the total number of recorded instances of a behaviour (e.g. social 
204 learning) and an estimate of research effort (e.g. the number of papers published on that taxa; e.g.

205 Sol, et al., 2005; Reader, et al., 2011; Navarrete, et al., 2016; Ducatez, et al., 2020). However, the

206 choice of how to model the relationship between total number of recorded innovation or social

207 learning instances and research effort matters considerably when creating these residual rate

208 measures. For our data, the relationship between the total number of innovation or social learning

209 instances and research effort was non-linear. This made the choice of model structure non-trivial,

210 with different models proving difficult to justify over one another. Residuals from these models

211 additionally showed further structural issues, including failure to meet assumptions about

212 homoscedasticity. Thus, we opted to use binary measures, which allowed us to use data

213 imputation methods to minimize biases caused by under-studied taxa, statistically account for

214 potential biases associated with summarizing behavioural data at higher taxonomic levels, and

215 run simulations to address the assumptions underlying our analyses. We therefore assigned each

216 lineage or genus binary scores of 1 (presence of innovation or social learning) or 0 (absence of

217 innovation or social learning). Further considerations regarding the use of literature-based

218 estimates of behavioural flexibility can be found in the supplementary material.

220 Structural Proxies of Behavioural Flexibility

221 Literature-based evidence for behavioural flexibility across taxa has its limitations and so we

222 chose to also consider structural correlates of behavioural flexibility. It is widely thought that

223 particular brain regions are associated with flexible behaviour - particularly the neocortex (see,

224 e.g., Keverne, et al., 1996; Mikhalevich, et al., 2017) and cerebellum (Vandervert, 2003;

225 Vandervert, et al., 2007; Barton, 2012). Therefore, in addition to total brain size (relative to body 226 mass), we considered the sum of neocortical and cerebellar volumes relative to rest of total brain 
227 volume as another proxy for behavioural flexibility. Using both behavioural measures and

228 structural correlates of behavioural flexibility, we were able to compare the consistency of results

229 across different proxies for behavioural flexibility.

231 Lineage-level estimates for all brain measures were calculated by taking the geometric mean of

232 taxon volumes for each lineage. Brain volume relative to body size (hereafter 'relative brain

233 volume') was estimated by regressing lineage-level estimates of logarithmic endocranial volume,

234 in $\mathrm{cm}^{3}$, (ECV) (Powell, et al., 2017) (hereafter 'brain volume') as a function of logarithmic body

235 mass, in grams (Jones, et al., 2009), and retaining the residuals (Dunbar \& Schultz, 2007).

236 Notably, previous studies (e.g. Sayol, et al., 2019) have used phylogenetically corrected residuals

237 to account for an effect of body size when using similar brain size measures. However, for our

238 data, residuals obtained from phylogenetic models were skewed such that lineages with larger

239 body mass consistently had larger residual brain volumes (see Figure S2), and thus we opted to

240 use ordinary least square residuals that were more orthogonal to lineage body size. Consistent

241 with relative brain volume, neocortex and cerebellum volume relative to rest-of-brain volume

242 (hereafter 'relative neocortex and cerebellum volume') was estimated by taking the residuals

243 from a log-log regression of the combined neocortex and cerebellum volumes on the rest-of-

244 brain volumes (i.e. total brain volume minus neocortex and cerebellum volumes) taken from

245 Navarrete et al. (2018) and the compilation in DeCasien \& Higham (2019). Genus-level

246 estimates for structural proxies of flexibility were calculated by taking the mean of the lineage

247 estimates within each genus. Further details, and considerations regarding the use of structural

248 proxies of behavioural flexibility, and residual brain measures can be found in the supplementary

249 materials along with correlation coefficients for all predictor variables (Table S1). 
251 Phylogenetic signal of all predictor and response variables are reported in the supplementary 252 material (Table S2).

\section{Analysis}

255 All analyses used R version 3.6.3 (R Core Team, 2020).

\section{Trait Imputation}

258 While we collected data for our predictor variables from the most comprehensive datasets and

259 compilations available, research biases and the persistent reassessment of primate taxonomy has

260 resulted in inconsistent data coverage across lineages, and there were still many lineages that

261 were missing data (see Figure S3). To maximize our evolutionary inferences on diversification

262 and allow for the inclusion of data-poor lineages, we chose to impute missing predictor variables

263 using phylogenetic imputation methods (see supplementary material for details and reports of

264 predictive accuracy from model cross-validation; Table S3 and Figure S4). Data on relative

265 neocortex and cerebellum volume were sparse and unevenly distributed across the phylogeny

266 (82.4\% of lineages missing data; Table S3), making it infeasible to reliably impute missing

267 values. We thus only ran models of relative neocortex and cerebellum volume on the original,

268 non-imputed dataset. All of the regressions we report below were repeated for the original, non-

269 imputed datasets (see Results and the supplementary material Tables S4 to S6) and except as

270 noted gave similar results. 


\section{Predictors of Diversification}

273 To assess the relationship between our measures of behavioural flexibility and diversification

274 rate at the lineage-level (Taxa per Lineage Diversification Rate) we used phylogenetic

275 generalized least squares (PGLS) regressions implemented using the "gls" function in the nlme

276 package (Pinheiro, et al., 2020), including the 10kTrees consensus tree for our 239 defined

277 lineages as the phylogenetic backbone. PGLS is a common regression method used to investigate 278 evolutionary associations while accounting for the fact that closely-related lineages tend to be

279 similar (e.g. in body size, life history and ecology; see Freckleton, et al., 2002). Continuous data

280 were log-transformed, centred with respect to the mean, and scaled by 2 standard deviations in

281 all models for both the lineage and genus-level analyses to make effect sizes comparable to those

282 reported for binary variables (Gelman, 2008). After imputing missing values, our dataset

283 contained 54 lineages scored as having evidence of social learning (scored as 1) and 28 lineages

284 scored as having evidence of technical innovation (scored as 1). Wyles et al. (1983) predicted

285 accelerated evolution in species with a dual capacity for innovation and social propagation of

286 new behaviours, therefore, we also tested a combined measure of technical innovation and social

287 learning. In this combined measure 26 lineages with the presence of both behaviours were scored

288 as 1, and those exhibiting only one or neither behaviour were scored as 0.

290 To assess the relationship between our measures of behavioural flexibility and diversification

291 rate deeper in the tree we repeated the same analysis at the genus-level using two different

292 estimates of diversification rate: Taxa per Genus Diversification Rate and the more conservative 
293 Lineage per Genus Diversification Rate. After imputing missing values, our dataset of 60 genera

294 contained 21 genera scored as having evidence of social learning, 9 genera scored as having

295 evidence of technical innovation and 8 genera with evidence of both behaviours.

297 Body mass and attendant life history traits have been predicted to impact diversification rates in

298 some taxa, albeit with conflicting results (see, e.g., Cardillo, et al., 2003; Paradis, 2005;

299 Fontanillas, et al., 2007; Thomas, et al., 2010), and body mass is closely correlated with many

300 primate life history traits (e.g. age at first reproduction, maximum lifespan; Charnov \& Berrigan,

301 1993; Purvis, et al., 2003; Street, et al., 2017). To examine whether our results could stem from

302 confounding effects of body mass and its correlates, we ran PGLS analyses to test body mass as

303 an independent predictor of our diversification rate measures. Results from these tests were non-

304 significant across all measures of diversification (see supplementary material Tables S4 to S6).

306 We re-ran all PGLS analyses reported above after eliminating the great apes, which are

307 characterized by slow life histories, which may counteract the evolutionary effects of

308 behavioural flexibility by slowing their evolutionary response time (Price, et al., 2003). For tests

309 that proved significant, we then reran the same test 100 times over a sample of 1000 trees from

310 the tree block available in 10kTrees (Arnold, et al., 2010) to confirm results were consistent after

311 accounting for phylogenetic uncertainty. 
314 Genera were considered behaviourally flexible if any of their daughter lineages had evidence of

315 innovation or social learning, which potentially introduces a statistical bias if more lineage-rich

316 genera (which will generally have higher diversification rates) are more likely by chance to

317 include at least one lineage that expresses technical innovation or social learning. To account for

318 this possible bias, we simulated the neutral evolution of technical innovation and social learning

319 across the primate phylogeny 1000 times using the symmetrical rate Mk model of discrete trait

320 evolution (Lewis, 2001). We opted to model the evolution of these behaviours under a

321 symmetrical model of trait evolution since with smaller datasets such as ours, there is little power

322 to prefer asymmetric models (Mooers \& Schluter, 1999). From these stochastic distributions of

323 the two traits, we repeated our analyses of diversification rate and created a distribution of

324 expected effect sizes under a null evolutionary scenario. More diverse genera may also be more

325 likely, by chance, to have well-studied lineages, which could create a bias toward observing the

326 presence of innovation or social learning in diverse genera (Ducatez \& Lefebvre, 2014).

327 Although data did not suggest that lineage-rich clades were more likely to have intensely

328 investigated lineages in our dataset (see Figures S5 and S6), we nonetheless opted to take

329 research effort into account in our analysis and expanded upon our first simulation to consider a

330 scenario where the presence of technical innovation or social learning may go unobserved if

331 insufficient research effort was directed at a lineage. We took the 1000 simulations of neutral

332 evolution of technical innovation and social learning, and then randomly 'evolved' research

333 effort for each simulation, independently of the evolution of technical innovation and social

334 learning. We then converted the presence of these behaviours to absences in our simulated

335 datasets if a lineage's 'evolved' research effort was below the minimum threshold of studies for

336 a lineage with observed technical innovation (as it contained the higher research effort threshold 
337 than social learning; Figure S7). By repeating our analyses of diversification rate with these new

338 simulated datasets where lineages with low research effort were assigned 'hidden states', we

339 were able to see if a bias towards having better studied lineages in diverse genera could drive a

340 positive association between behavioural proxies of flexibility and diversification rate by chance

341 and independent of biological mechanisms. Importantly, this simulation makes the assumption

342 that research effort and behavioural flexibility are independent; if this is not true such that

343 behaviourally flexible lineages attract research effort (see discussion in the 'Research Effort

344 Bias' section of the supplementary material) then the results of this simulation would actually be

345 conservative. We detail both simulations, along with considerations about using research effort

346 as a covariate in binary models (Ducatez, et al., 2020), in the supplementary material.

348 Ethical Note

349 This research was comparative and was based on data available in previously published

350 literature.

352 RESULTS:

\section{Lineage-Level Predictors of Diversification}

354 We found no support for an association between behavioural flexibility and diversification rates

355 when testing our measures of behavioural flexibility at the lineage-level (results summarized in

356 Figures 1 and S8; Table S4). Social learning $(p=0.171)$, technical innovation $(p=0.792)$, the

357 combined presence of technical innovation and social learning $(p=0.979)$, relative brain volume 
$358(p=0.215)$, relative neocortex and cerebellum volume $(p=0.664)$, and body mass $(p=0.764$

359 Table S4) did not exhibit noteworthy associations with Taxa per Lineage Diversification Rate in 360 either direction.

Genus-Level Predictors of Diversification

363 Technical innovation $(p=0.577)$, social learning $(p=0.442)$, the combined presence of technical

364 innovation and social learning $(\mathrm{p}=0.411)$, relative neocortex and cerebellum volume $(\mathrm{p}=0.930)$,

365 and body mass $(\mathrm{p}=0.204)$ did not exhibit noteworthy associations with Taxa per Genus

366 Diversification Rate (Figures 1 and S8; Table S5). Relative brain volume was insignificantly ( $\mathrm{p}=$

367 0.108), but positively, related to diversification rate, and this relationship became significant

368 when we considered the non-imputed dataset $(p=0.004)$.

370 In contrast to the other diversification measures, Lineage per Genus Diversification Rate was

371 positively associated with all three behavioural measures of behavioural flexibility (results

372 summarized in Figures 1 and S8; Table S6). Genera with records of technical innovation were

373 shown to have a faster mean Lineage per Genus Diversification Rate $\left(0.136\right.$ lineages my $\left.{ }^{-1}\right)$ than

374 those with no reports $\left(0.070\right.$ lineages $\left.\mathrm{my}^{-1} ; \beta[95 \% \mathrm{CI}]=0.066[0.002-0.130] ; \mathrm{p}=0.048\right)$. Genera

375 with records of social learning similarly exhibited a faster mean Lineage per Genus

376 Diversification Rate $\left(0.137\right.$ lineages $\left.\mathrm{my}^{-1}\right)$ than those without $\left(0.049\right.$ lineages $\mathrm{my}^{-1} ; \beta[95 \% \mathrm{CI}]$

$377=0.088$ [0.043-0.132]; $<<0.001)$. Genera with reports of both technical innovation and social

378 learning also exhibited a faster mean Lineage per Genus Diversification Rate (0.153 lineages my

$\left.379^{1}\right)$ compared to those with evidence for only one or neither behaviour $\left(0.068\right.$ lineages my $^{-1} ; \beta$ 
$380[95 \% \mathrm{CI}]=0.085[0.018-0.151] ; \mathrm{p}=0.015)$. These results were consistent with results run over a

381 sample of 1000 trees from the posterior distribution, i.e. "tree block", available from 10kTrees

382 (Arnold, et al., 2010), indicating results were not biased by our choice of phylogeny (Figure S9).

383 Based on our simulation testing for a lineage-richness sampling bias, we found that these effect

384 sizes were unlikely to be due to chance alone for technical innovation $(p=0.028)$, social learning

$385(\mathrm{p}=0.002)$ and the combined presence of both behaviours $(\mathrm{p}=0.008)$ (see Figure S10 and

386 supplementary results). After expanding our simulation to consider a research effort bias,

387 significance of these effects remained for social learning $(p=0.019)$ and its combined presence

388 with technical innovation $(\mathrm{p}=0.027)$, but the effect of technical innovation alone was no longer

389 nominally significant ( $\mathrm{p}=0.109)$ (see Figure S11 and supplementary results). No structural

390 proxies of behavioural flexibility shared notable associations with Lineage per Genus

391 Diversification Rate (Figures 1 and S8; Table S6).

392

393 Results of all PGLS analyses reported above were qualitatively consistent after removing great

394 apes (see supplementary material Tables S7 to S9). 


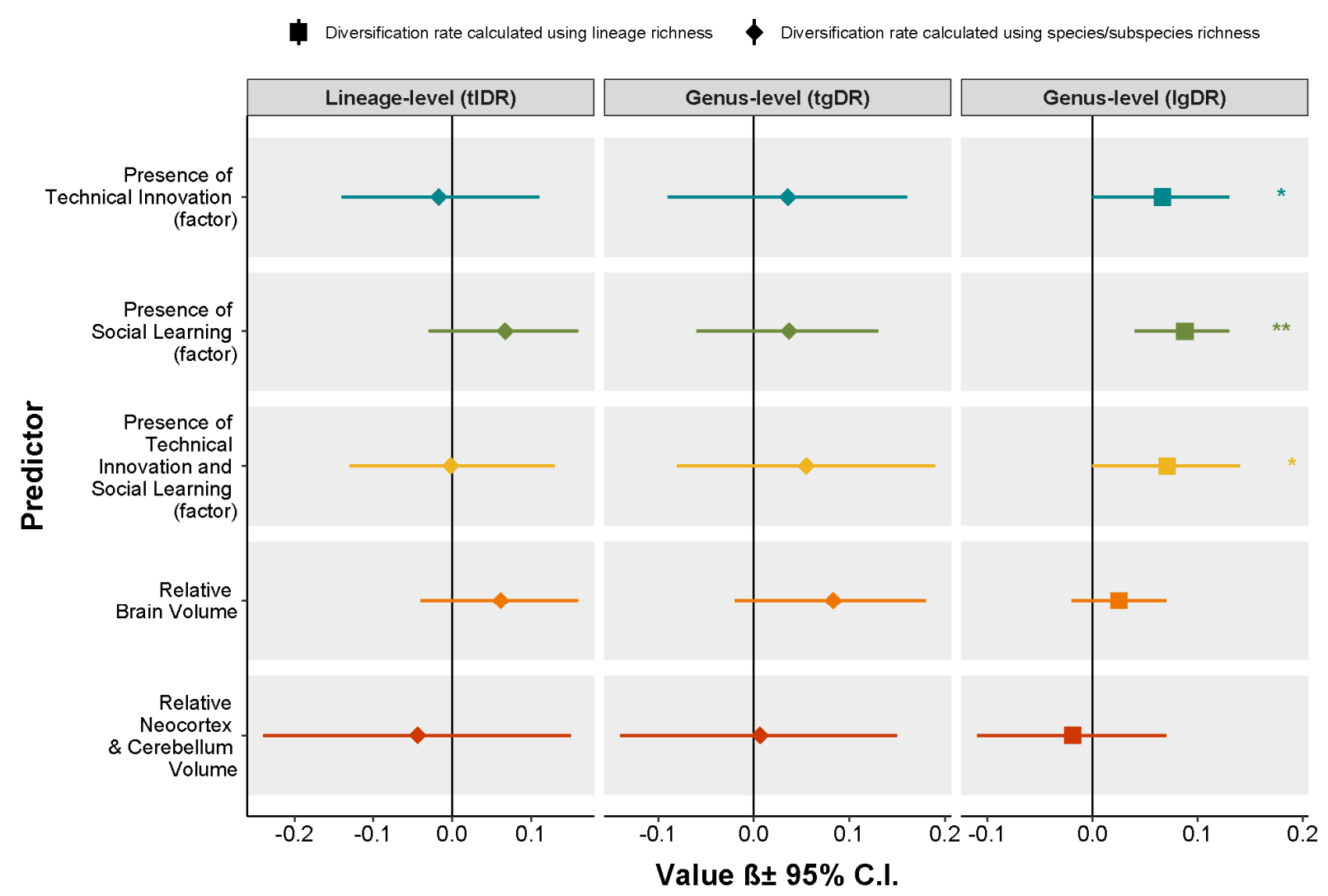

397 Figure 1: Results from PGLS analyses showing the association of proxies of behavioural

398 flexibility with Taxa per Lineage Diversification Rate (DR), Taxa per Genus Diversification

399 Rate and Lineage per Genus Diversification Rate across primates. 95\% confidence intervals are

400 represented by horizontal lines around the associated value. Diamonds indicate diversification

401 rates estimated with species/subspecies richness, and squares indicate diversification rates

402 estimated with lineage richness. Results presented include imputed data when available and brain

403 measures were standardized $(\ln \mathrm{x} /(2 \mathrm{sd}))$. Significance indicated as: $+\mathrm{P} \leq 0.1 ; * \mathrm{P} \leq 0.05 ; * * \mathrm{P} \leq 0.01$. 
406 We find little to no compelling support for an association between our proxy measures of

407 behavioural flexibility and recent primate diversification rates of young species and subspecies,

408 however, we do find some evidence supporting a positive association when looking at the

409 diversification of older primate lineages. This pattern could be explained in several ways, and

410 interpretation of these results depend on the assumptions one makes about our different measures

411 of diversification rate.

412

413 One benefit of our study design is that it allowed us to consider taxa that are commonly

414 overlooked (i.e. subspecies and species omitted from higher order phylogenies), many of which

415 likely represent very recent splitting events. The weak associations with behavioural flexibility

416 that we observed at shallower time scales could be explained in several ways. On one hand, this

417 pattern could reflect biases in describing taxa (species and subspecies) among groups. While our

418 time cut-off, and use of both species and subspecies, mitigates against biases associated with the

419 elevation of subspecies under the PSC, if less flexible species are more likely to have a larger

420 number of taxa described overall (e.g. based on regional biases in designating species or

421 subspecies that covary with locations of inflexible clades) then this could obscure underlying

422 biological patterns. If this is the case, then behavioural flexibility may indeed enhance

423 diversification by acting on speciation, and we observe stronger associations when ignoring

424 shallow splits because our diversification rate metric calculated using lineage-richness is less

425 biased by this phenomenon. On the other hand, if we take our results at face value, a pattern of

426 stronger associations deeper in the phylogeny could indicate that time plays an even larger role

427 in the relationship between behavioural flexibility and diversification than previously suggested

428 by Duckworth (2009). 
430 Under Mayr's 'ephemeral speciation model' (Mayr, 1963; Rosenblum, et al., 2012) and the

431 related 'ephemeral divergence hypothesis' (Futuyma, 1979; 2010), divergence can occur rapidly

432 and often, but many newly diversifying lineages do not persist, instead being eradicated via

433 extinction or 'reabsorption' by hybridization (see, e.g., Rosenblum, et al., 2012). It is possible

434 that a number of the described species and subspecies used here (from the IUCN/SSC Primate

435 Specialist Group, 2018) represent such ephemeral diversification events, especially considering

436 that the PSC has facilitated the splitting of very closely related populations. If so, our results

437 could be explained if behavioural flexibility buffers against extinction through behavioural shifts.

438 Behavioural flexibility would then promote diversification through bolstering lineage persistence

439 rather than the rate of splitting; and would be revealed when comparing the accumulation of

440 lineages that have escaped extinction (i.e. looking deeper in the tree). This would be

441 complementary to findings from Arbour \& Santana (2017), who show that decreased extinction

442 preceded a shift to increased evolutionary rates in the most speciose primate family

443 (Cercopithecidae), and with evidence suggesting behavioural flexibility is beneficial for

444 population persistence in birds (e.g. Shultz, et al., 2005; Rossmanith, et al., 2006; Sol, et al.,

445 2007, Ducatez, et al., 2020). Under this scenario, the positive associations we find are not the

446 result of behavioural flexibility enhancing evolutionary rates by facilitating divergence events,

447 and instead indicate that extinction resistance may be an important mechanism linking

448 behavioural flexibility and primate diversification. Our results stand in contrast with consistent

449 reports of behavioural flexibility enhancing diversification of birds even at shallow phylogenetic

450 depths (Nicolakakis, et al., 2003; Sol, et al., 2005; Sayol, et al., 2019). 
452 A potential explanation for differing effects of behavioural flexibility in birds versus primates

453 could be that the heightened dispersal capabilities (i.e. flight) of birds makes behavioural

454 flexibility particularly beneficial for these taxa when it comes to establishing in new

455 environments. This could cause behavioural flexibility to enhance bird speciation via increased

456 success in dispersal events while also buffering against extinction - leading to the strong effects

457 that have been observed on their net rates of evolution (Nicolakakis, et al., 2003; Sol, 2003; Sol,

458 et al., 2005; Sayol, et al., 2019). Comparatively, primates have much more limited dispersal

459 capabilities and thus behavioural flexibility may buffer against extinction while having little

460 effect on promoting allopatric establishment and subsequent speciation. If true, it is possible that

461 behavioural flexibility affects evolutionary trajectories via different mechanisms in these clades.

463 Directly testing whether behavioural flexibility buffers against extinction may be difficult

464 because measuring extinction rates at macroevolutionary scales is notoriously imprecise

465 (Rabosky, 2010; Louca \& Pennell, 2020). Future studies could test proxies of primate

466 behavioural flexibility against estimates of contemporary extinction vulnerability (see, e.g.,

467 Nicolakakis, et al., 2003; Ducatez, et al., 2020). Another prediction concerns the rate of genetic

468 divergence within flexible versus less flexible, recent clades (e.g. subspecies within species): our

469 conjecture would be that these would not be different, because flexibility does not lead to

470 increased divergence over the short term.

471

472 Notably, not all of our results are consistent with a significantly positive association between

473 behavioural flexibility and diversification of older primate lineages. While the non-significant

474 association between Lineage per Genus Diversification Rate and neocortex and cerebellum 
475 volume might be explained by data limitations (discussed below), the non-significant association

476 with relative brain volume illustrates that not all proxies of behavioural flexibility capture the

477 same thing. While relative brain volume is a known structural correlate of measures of primate

478 and avian behavioural flexibility (Lefebvre, et al., 2004), the brain has many functions and thus

479 is a less direct measure of behavioural flexibility than behavioural measures. Measures of brain

480 size have been linked to diversification rate in birds (Nicolakakis, et al., 2003; Sol, et al., 2005;

481 Sayol, et al., 2019), but notably the sample for birds is much larger, and is likely to contain a

482 broader range of variation, which would make it easier to detect a brain size effect. Additionally,

483 results of our simulation accounting for potential research effort biases indicate that a positive

484 association between technical innovation and diversification rate in primate genera could be

485 driven by a research effort bias. However, this simulation assumes that research effort and

486 behavioural flexibility are independent and, if instead research effort tends to be directed toward

487 groups where innovation is expected, then this research bias may actually have a low

488 contribution to the observed relationship between technical innovation and diversification rate.

489 Thus, our results paint a complicated picture, and emphasize the need to compare methodologies

490 and measures, and avoid a focus on single explanations for complex phenomena.

492 Our results provide some support for primate behavioural flexibility being associated with older

493 as opposed to more recent diversification events, however, there are several caveats on the limits

494 of our inferences. First, one measure thought to be associated with behavioural flexibility -

495 neocortex and cerebellum volume - had very little data available for lineages reported as

496 inflexible (e.g. among lemurs, tarsiers, titis and sakis). This meant our ability to test this measure

497 as a driver of diversification was limited. Additionally, to examine the relationship between 
different measures of behavioural flexibility and diversification rate, we had to run multiple tests,

499 increasing the likelihood of observing Type I error. Our study is also limited by the lineages

500 present in the phylogeny. Available primate phylogenies, (e.g. Arnold, et al., 2010; Upham, et

501 al., 2019b) do not reflect the most recent taxon lists, and such limited taxonomic scope prevented

502 us from using diversification rate estimates that require a fully-resolved tree (e.g. the DR

503 measures from Jetz, et al., 2012). Lastly, many factors will contribute to true diversification, and

504 although we test two (e.g. body mass and a "great ape effect"; see supplemental results), there are

505 many biological and ecological factors (e.g. geographic discontinuities, habitat affiliations, or

506 presence on islands) yet to be explored that could mediate any relationship between behavioural

507 flexibility and primate diversification rate.

509 While our results provide some evidence for the hypothesis that behavioural flexibility drives

510 diversification of primate lineages, they raise important questions about its underlying

511 mechanisms. Importantly, our results are not consistent in supporting a significant association

512 between behavioural flexibility and diversification rate, and depend on the proxy measures

513 employed. The positive associations we do find may point to behavioural flexibility dampening

514 extinction of young primate lineages, rather than accelerating diversification via splitting of

515 behaviourally shifted individuals/populations. Thus, expanding tests of whether and how

516 behavioural flexibility is associated with diversification to other taxa may help us interpret the

517 results we report here.

518

519 ACKNOWLEDGEMENTS: This work was supported by the Natural Sciences and Engineering

520 Research Council of Canada (NSERC) Discovery Grants Program (SMR and AOM, grants 
521 \#2017-04720 and \#2019-04950) and the Canada Foundation for Innovation (SMR, grant

522 \#29433). MJAC was supported by awards from the Biodiversity, Ecosystem Services, and

523 Sustainability (BESS) program and McGill University. We thank the Reader and Guigueno labs,

524 Brian Leung, and Hans Larsson at McGill, William Wcislo, and the Crawford Lab for

525 Evolutionary Studies at SFU for feedback on study design and interpretation, as well as Isabella

526 Capellini and three anonymous reviewers for comments on previous versions.

527

528 Data and code will be made available through Dryad or a similar repository.

532 REFERENCES:

533 Anderson, R. W. (1995). Learning and evolution: A quantitative genetics approach. Journal of

534 Theoretical Biology, 175: 89-101.

535 Ancel, L. W. (1999). A quantitative model of the Simpson-Baldwin effect. Journal of

536 Theoretical Biology, 196: 197-209.

537 Ancel, L. W. (2000). Undermining the Baldwin expediting effect: does phenotypic plasticity

538 accelerate evolution? Theoretical Population Biology, 58: 307-319.

539 Arbour, J. H., \& Santana, S. E. (2017). A major shift in diversification rate helps explain

540 macroevolutionary patterns in primate species diversity. Evolution, 71: 1600-1613.

541 Arnold, C., Matthews, L. J., \& Nunn, C. L. (2010). The 10kTrees website: a new online resource 542 for primate phylogeny. Evolutionary Anthropology, 19: 114-118. 
543 Audet, J. N., \& Lefebvre, L. (2017). What's flexible in behavioural flexibility? Behavioural $544 \quad$ Ecology, 28: 943-947.

545 Badgley, C. (2010). Tectonics, topography, and mammalian diversity. Ecography, 33: 220-231.

546 Baldwin, J. M. (1902). Development and evolution. MacMillan, New York.

547 Barton, R. A. (2012). Embodied cognitive evolution and the cerebellum. Philosophical

548 Transactions of the Royal Society B: Biological Sciences, 367: 2097-2107.

549 Bateson, P. (1988). The active role of behaviour in evolution. In M. -W. Ho \& S. W. Fox (Eds.), 550 Evolutionary processes and metaphors (p. 191-207). John Wiley and Sons, Ltd., New York.

551 Behera, N., \& Nanjundiah, V. (1995). An investigation into the role of phenotypic plasticity in 552 evolution. Journal of Theoretical Biology, 172: 225-234.

553 Bogert, C. M. (1949). Thermoregulation in reptiles, a factor in evolution. Evolution, 3: 195-211.

554 Cardillo, M., Huxtable, J. S., \& Bromham, L. (2003). Geographic range size, life history and 555 rates of diversification in Australian mammals. Journal of Evolutionary Biology, 16: 282556288

557 Charnov, E. L., \& Berrigan, D. (1993). Why do female primates have such long lifespans and so 558 few babies? Or life in the slow lane. Evolutionary Anthropology, 1: 191-194.

559 DeCasien, A. R., \& Higham, J. P. (2019). Primate mosaic brain evolution reflects selection on $560 \quad$ sensory and cognitive specialization. Nature Ecology \& Evolution, 3: 1483-1493.

561 Ducatez, S., Clavel, J., \& Lefebvre, L. (2015). Ecological generalism and behavioural 562 innovation in birds: technical intelligence or the simple incorporation of new foods? $563 \quad$ Journal of Animal Ecology, 84: 79-89. 
564 Ducatez, S., \& Lefebvre, L. (2014). Patterns of research effort in birds. PLoS One, 9: e89955.

565 Ducatez, S., Sol, D., Sayol, F., \& Lefebvre, L. (2020). Behavioural plasticity is associated 566 with reduced extinction risk in birds. Nature Ecology \& Evolution, 4: 788-793.

567 Duckworth, R. A. (2009). The role of behaviour in evolution: a search for mechanism.

$568 \quad$ Evolutionary Ecology, 23: 513-531.

569 Dunbar, R. I., \& Shultz, S. (2007). Understanding primate brain evolution. Philosophical

570 Transactions of the Royal Society B: Biological Sciences, 362: 649-658.

571 Fontanillas, E., Welch, J. J., Thomas, J. A., \& Bromham, L. (2007). The influence of body size 572 and net diversification rate on molecular evolution during the radiation of animal phyla. $573 \quad$ BMC Evolutionary Biology, 7: 95.

574 Foote, A. D., Vijay, N., Ávila-Arcos, M. C., Baird, R. W., Durban, J. W., Fumagalli, M., ... \& 575 Wolf, J. B. W. (2016). Genome-culture coevolution promotes rapid divergence of killer $576 \quad$ whale ecotypes. Nature Communications, 7: 11693.

577 Freckleton, R. P., Harvey, P. H., \& Pagel, M. (2002). Phylogenetic analysis and comparative 578 data: a test and review of evidence. The American Naturalist, 160: 712-726.

579 Frey, M. A. (2010). The relative importance of geography and ecology in species diversification: 580 evidence from a tropical marine intertidal snail (Nerita). Journal of Biogeography, 37: 1515$581 \quad 1528$.

582 Futuyma, D. J. (1979). Evolutionary biology. Sinauer Associates, Sunderland.

583 Futuyma, D. J. (2010). Evolutionary constraint and ecological consequences. Evolution, 64: $584 \quad 1865-1884$. 
585 Gelman, A. (2008). Scaling regression inputs by dividing by two standard deviations. Statistics

586 in Medicine, 27: 2865-2873.

587 Gonzalez-Voyer, A., González-Suárez, M., Vilà, C., \& Revilla, E. (2016). Larger brain size

588 indirectly increases vulnerability to extinction in mammals. Evolution, 70: 1364-1375.

589 Grant, P. R., \& Grant, B. R. (2008). How and why species multiply: the radiation of Darwin's

$590 \quad$ finches. Princeton University Press, Princeton.

591 Groves, C. P. (2014). Primate taxonomy: inflation or real? Annual Review of Anthropology, 43:

$592 \quad 27-36$.

593 Heard, S. B., \& Hauser, D. L. (1995). Key evolutionary innovations and their ecological

594 mechanisms. Historical Biology, 10: 151-173.

595 Hinton, G. E., \& Nowlan, S. J. (1987). How learning can guide evolution. Complex Systems, 1:

$596 \quad 495-502$.

597 Honacki, J. H., Kinman, K. E., \& Koeppl, J. W. (1982). Mammal species of the world: a

598 taxonomic and geographic reference. Allen Press, Inc. and Association of Systematic

599 Collections, Lawrence.

600 Huey, R. B., Hertz, P. E., \& Sinervo, B. (2003). Behavioural drive versus behavioural inertia in 601 evolution: a null model approach. The American Naturalist, 161: 357-366.

602 Isaac, N. J., Jones, K. E., Gittleman, J. L., \& Purvis, A. (2005). Correlates of species richness in 603 mammals: body size, life history, and ecology. The American Naturalist, 165: 600-607.

604 Isaac, N. J., Mallet, J., \& Mace, G. M. (2004). Taxonomic inflation: its influence on 605 macroecology and conservation. Trends in Ecology \& Evolution, 19: 464-469. 
606 IUCN/SSC Primate Specialist Group. (2018). Retrieved from http://www.primate-

607 sg.org/red_list_threat_status/[accessed 10 November 2018].

608 Jetz, W., Thomas, G. H., Joy, J. B., Hartmann, K., \& Mooers, A. O. (2012). The global diversity 609 of birds in space and time. Nature, 491: 444-448.

610 Jones, K. E., Bielby, J., Cardillo, M., Fritz, S. A., O'Dell, J., Orme, C. D. L., ... \& Connolly, C. 611 (2009). PanTHERIA: a species-level database of life history, ecology, and geography of 612 extant and recently extinct mammals: Ecology, 90: 2648-2648.

613 Louca, S., \& Pennell, M. W. (2020). Extant timetrees are consistent with a myriad of 614 diversification histories. Nature, 580: 502-505.

615 Keverne, E. B., Martel, F. L., \& Nevison, C. M. (1996). Primate brain evolution: genetic and 616 functional considerations. Proceedings of the Royal Society B: Biological Sciences, 263: $617 \quad 689-696$.

618 Kozak, K. H., \& Wiens, J. J. (2010). Accelerated rates of climatic-niche evolution underlie rapid 619 species diversification. Ecology Letters, 13: 1378-1389.

620 Lachlan, R. F., \& Servedio, M. R. (2004). Song learning accelerates allopatric speciation.

$621 \quad$ Evolution, 58: 2049-2063.

622 Lapiedra, O., Sol, D., Carranza, S., \& Beaulieu, J. M. (2013). Behavioural changes and the 623 adaptive diversification of pigeons and doves. Proceedings of the Royal Society B:

624 Biological Sciences, 280: 20122893.

625 Lefebvre, L., Reader, S. M., \& Sol, D. (2004). Brains, innovations and evolution in birds and 626 primates. Brain, Behaviour and Evolution, 63: 233-246. 
627 Lewis, P. O. (2001). A likelihood approach to estimating phylogeny from discrete morphological 628 character data. Systematic Biology, 50: 913-925.

629 Lewontin, R. C. (1983). Gene, organism and environment. In D. S. Bendall (Eds.), Evolution 630 from molecules to men (p. 273-285). Cambridge University Press, New York.

631 Losos, J. B., Schoener, T. W., \& Spiller, D. A. (2004). Predator-induced behaviour shifts and 632 natural selection in field-experimental lizard populations. Nature, 432: 505-508.

633 Magallon, S., \& Sanderson, M. J. (2001). Absolute diversification rates in angiosperm clades. Evolution, 55: 1762-1780.

635 Mayr, E. (1963). Animal species and evolution. Belknap Press, Cambridge.

636 Mikhalevich, I., Powell, R., \& Logan, C. (2017). Is behavioural flexibility evidence of 637 cognitive complexity? How evolution can inform comparative cognition. Interface $638 \quad$ Focus, 7: 20160121.

639 Mitchell, J. S., \& Rabosky, D. L. (2017). Bayesian model selection with BAMM: effects of 640 the model prior on the inferred number of diversification shifts. Methods in Ecology $641 \quad$ and Evolution, 8: 37-46.

642 Mooers, A. Ø., \& Schluter, D. (1999). Reconstructing ancestor states with maximum likelihood: 643 support for one-and two-rate models. Systematic Biology, 48: 623-633.

644 Muñoz, M. M., \& Losos, J. B. (2018). Thermoregulatory behaviour simultaneously promotes 645 and forestalls evolution in a tropical lizard. The American Naturalist, 191: E15-E26.

646 Navarrete, A. F., Blezer, E. L., Pagnotta, M., de Viet, E. S., Todorov, O. S., Lindenfors, P., ... 647 \& Reader, S. M. (2018). Primate brain anatomy: new volumetric MRI measurements 
Navarrete, A. F., Reader, S. M., Street, S. E., Whalen, A., \& Laland, K. N. (2016). The coevolution of innovation and technical intelligence in primates. Philosophical Transactions of the Royal Society B: Biological Sciences, 371: 20150186.

653 Nicolakakis, N., Lefebvre, L. \& Sol, D. (2003). Behavioural flexibility predicts species richness in birds, but not extinction risk. Animal Behaviour, 65: 445-452.

655 Odling-Smee, F. J., Laland, K. N., \& Feldman, M. W. (2003). Niche construction: the neglected process in evolution. Princeton University Press, Princeton. innovations drive the relationship between innovativeness and residual brain size in birds. Animal Behaviour, 78: 1001-1010.

662 Pelletier, F., Garant, D., \& Hendry, A. P. (2009). Eco-evolutionary dynamics. Philosophical Transactions of the Royal Society B: Biological Sciences, 364: 1483-1489.

664 Pfennig, D. W., Wund, M. A., Snell-Rood, E. C., Cruickshank, T., Schlichting, C. D., \& 665 Moczek, A. P. (2010). Phenotypic plasticity's impacts on diversification and speciation. Trends in Ecology \& Evolution, 25: 459-467. 
667 Pinheiro, J., Bates, D., DebRoy, S., Sarkar, D., \& R Core Team. (2020). Linear and nonlinear

668 mixed effects models. R package version 3.1-144. https://CRAN.R-

$669 \quad$ project.org/package $=$ nlme.

670 Powell, L. E., Isler, K., \& Barton, R. A. (2017). Re-evaluating the link between brain size and

671 behavioural ecology in primates. Proceedings of the Royal Society B: Biological

$672 \quad$ Sciences, 284: 20171765.

673 Price, T. (2008). Speciation in birds. Roberts and Company, Greenwood Village.

674 Price, T. D., Qvarnström, A., \& Irwin, D. E. (2003). The role of phenotypic plasticity in

675 driving genetic evolution. Proceedings of the Royal Society B: Biological Sciences, $676 \quad 270: 1433-1440$.

677 Purvis, A., Webster, A. J., Agapow, P. M., Jones, K. E., \& Isaac, N. J. (2003). Primate life 678 histories and phylogeny. In P. M. Kappeler \& M. E. Pereira (Eds.), Primate life 679 histories and socioecology (p. 25-40). University of Chicago Press, Chicago.

680 R Core Team. (2020). R: A Language and environment for statistical computing. R

681 Foundation for Statistical Computing, Vienna, Austria. http://www.R-project.org/

682 Rabosky, D. L. (2009). Ecological limits and diversification rate: alternative paradigms to 683 explain the variation in species richness among clades and regions. Ecology Letters, $684 \quad 12: 735-743$.

685 Rabosky, D. L. (2010). Extinction rates should not be estimated from molecular phylogenies. $686 \quad$ Evolution, 64: 1816-1824. 
687 Raikow, R. J. (1986). Why are there so many kinds of passerine birds? Systematic Zoology, 688 35: $255-259$.

689 Reader, S. M., \& Biro, D. (2010). Experimental identification of social learning in wild animals. Learning \& Behaviour, 38: 265-283.

691 Reader, S. M., Hager, Y., \& Laland, K. N. (2011). The evolution of primate general and cultural intelligence. Philosophical Transactions of the Royal Society B, 366: 1017-

694 Reader, S. M., \& Laland, K. N. (2002). Social intelligence, innovation, and enhanced brain size in primates. Proceedings of the National Academy of Sciences, 99: 4436-4441.

696 Riesch, R., Barrett-Lennard, L. G., Ellis, G. M., Ford, J. K. B., \& Deecke, V. B. (2012). Cultural 697 traditions and the evolution of reproductive isolation: ecological speciation in killer whales? 698 Biological Journal of the Linnean Society, 106: 1-17.

699 Rosenblum, E. B., Sarver, B. A., Brown, J. W., Des Roches, S., Hardwick, K. M., Hether, T. D., $700 \quad$... \& Harmon, L. J. (2012). Goldilocks meets Santa Rosalia: an ephemeral speciation model 701 explains patterns of diversification across time scales. Evolutionary Biology, 39: 255-261.

702 Rossmanith, E., Grimm, V., Blaum, N., \& Jeltsch, F. (2006). Behavioural flexibility in the 703 mating system buffers population extinction: lessons from the lesser spotted woodpecker $704 \quad$ Picoides minor. Journal of Animal Ecology, 75: 540-548.

705 Rylands, A. B., \& Mittermeier, R. A. (2014). Primate taxonomy: species and conservation. Evolutionary Anthropology, 23: 8-10. 
707 Sayol, F., Lapiedra, O., Ducatez, S., \& Sol, D. (2019). Larger brains spur species diversification 708 in birds. Evolution, 73: 2085-2093.

709 Scott-Phillips, T. C., Laland, K. N., Shuker, D. M., Dickins, T. E., \& West, S. A. (2014). The 710 niche construction perspective. A critical appraisal. Evolution, 68: 1231-1243.

711 Shultz, S. B., Bradbury, R. L., Evans, K. D., Gregory, R., \& Blackburn, T. M. (2005). Brain size 712 and resource specialization predict long-term population trends in British birds. Proceedings 713 of the Royal Society B: Biological Sciences, 272: 2305-2311.

714 Snell-Rood, E. (2013). An overview of the evolutionary causes and consequences of behavioural 715 plasticity. Animal Behaviour, 85: 1004-1011.

716 Snell-Rood, E., Swanson, E., \& Jaumann, S. (2015). Insects as a model system to understand the 717 evolutionary implications of innovation. In A. B. Kaufman \& J. C. Kaufman (Eds.), Animal 718 creativity and innovation (p. 459-478). Academic Press, London.

719 Sol, D. (2003). Behavioural flexibility: a neglected issue in the ecological and evolutionary 720 literature? In S. M. Reader \& K. N. Laland (Eds.), Animal innovation (p. 63-82). Oxford $721 \quad$ University Press, Oxford.

722 Sol, D., \& Lefebvre, L. (2000). Behavioural flexibility predicts invasion success in birds 723 introduced to New Zealand. Oikos, 90: 599-605.

724 Sol, D., \& Price, T. D. (2008). Brain size and the diversification of body size in birds. The $725 \quad$ American Naturalist, 172: 170-177. 
726 Sol, D., Stirling, D. G., \& Lefebvre, L. (2005). Behavioural drive or behavioural inhibition in

727 evolution: subspecific diversification in holarctic passerines. Evolution, 59: 2669-

728 2677.

729 Sol, D., Székely, T., Liker, A., \& Lefebvre, L. (2007). Big-brained birds survive better in nature. Proceedings of the Royal Society B: Biological Sciences, 274: 763-769.

731 Springer, M. S., Meredith, R. W., Gatesy, J., Emerling, C. A., Park, J., Rabosky, D. L., ... \&

732 Fisher, C. A. (2012). Macroevolutionary dynamics and historical biogeography of primate diversification inferred from a species supermatrix. PLoS One, 7: e49521.

Stadler, T. (2011). Mammalian phylogeny reveals recent diversification rate shifts. Proceedings of the National Academy of Sciences, 108: 6187-6192.

Street, S. E., Navarrete, A. F., Reader, S. M., \& Laland, K. N. (2017). Coevolution of cultural intelligence, extended life history, sociality, and brain size in primates. Proceedings of the National Academy of Sciences, 114: 7908-7914.

739 Tattersall, I. (2007). Madagascar's lemurs: cryptic diversity or taxonomic inflation?

$740 \quad$ Evolutionary Anthropology, 16: 12-23.

741 Tebbich, S., Sterelny, K., \& Teschke, I. (2010). The tale of the finch: adaptive radiation and

742 behavioural flexibility. Philosophical Transactions of the Royal Society B: Biological Sciences, 365: 1099-1109.

744 Thomas, J. A., Welch, J. J., Lanfear, R., \& Bromham, L. (2010). A generation time effect on the rate of molecular evolution in invertebrates. Molecular Biology and Evolution, 27: $1173-1180$. 
747 Upham, N., Esselstyn, J. A., \& Jetz, W. (2019a). Ecological causes of uneven diversification

$748 \quad$ and richness in the mammal tree of life (Preprint). bioRxiv, 504803.

749 Upham, N. S., Esselstyn, J. A., \& Jetz, W. (2019b). Inferring the mammal tree: Species-level

750 sets of phylogenies for questions in ecology, evolution, and conservation. PLoS

751 Biology, 17: 1-44.

752 Vandervert, L. (2003). The neurophysiological basis of innovation. In L. Shavinina (Eds.), 753 The international handbook on innovation (p. 17-30). Elsevier Science, Oxford.

754 Vandervert, L. R., Schimpf, P. H., \& Liu, H. (2007). How working memory and the

755 cerebellum collaborate to produce creativity and innovation. Creativity Research

$756 \quad$ Journal, 19: 1-18.

757 Wcislo, W. T. (1989). Behavioural environments and evolutionary change. Annual Review of $758 \quad$ Ecology and Systematics, 20: 137-169.

759 Weber, E. U., Shafir, S., \& Blais, A.-R. (2004). Predicting risk sensitivity in humans and lower

760 animals: risk as variance or coefficient of variation. Psychological Review, 111: 430-445.

761 West-Eberhard, M. J. (1989). Phenotypic plasticity and the origins of diversity. Annual review of

762 Ecology and Systematics, 20: 249-278.

763 West-Eberhard, M. J. (2003). Developmental plasticity and evolution. Oxford University

$764 \quad$ Press, Oxford.

765 Wiens, J. J. (2017). What explains patterns of biodiversity across the Tree of Life? New

766 research is revealing the causes of the dramatic variation in species numbers across

767 branches of the Tree of Life. BioEssays, 39: 1600128. 
768 Williams, C. B. (1964). Patterns in the balance of nature and related problems of quantitative ecology. Academic Press, London \& New York.

770 Willis, J. C. (1922). Area and age: A study of geographical distribution and origin in species. Cambridge University Press, Cambridge.

772 Wilson, A. C. (1985). The molecular basis of evolution. Scientific American, 253: 164-173.

773 Wyles, J. S., Kunkel, J. G., \& Wilson, A. C. (1983). Birds, behaviour, and anatomical

774 evolution. Proceedings of the National Academy of Sciences, 80: 4394-4397.

775 Yeh, P. J. (2004). Rapid evolution of a sexually selected trait following population

776 establishment in a novel habitat. Evolution, 58: 166-174.

777 Yeh, P. J., \& Price, T. D. (2004). Adaptive phenotypic plasticity and the successful colonization 778 of a novel environment. The American Naturalist, 164: 531-542. 